\title{
Simulated Annealing with Gaussian Probability Density Function for Transmission Expansion Planning
}

\author{
Phillipe Gomes \\ Department of Electrical and Computer Engineering, Faculty of Engineering, University of \\ Porto, Porto, Portugal (phillipe.gomes@fe.up.pt)
}

\begin{abstract}
Simulated Annealing (SA) is a powerful tool for optimization problems that have several local optima. This tool has the ability to escape from a local optima accepting relatively bad solutions for a period and searching for good solutions in your neighborhood. This paper describes the use of SA based on Gaussian Probability Density Function as a decision support criteria in resolution of Transmission Expansion Planning (TEP) problem. This method consists in starting from an initial solution with all possible circuits added and over the iterations removing, replacing or adding new circuits. The method proved to be a reasonable computational effort and proved able to find optimal values known in the literature.

Subject Headings. Electrical engineering, Planning, Optimization.

Author Keywords. Transmission Expansion Planning, Simulated Annealing, Local Search, Probability Density Function.
\end{abstract}

\section{Introduction}

Electric network expansion may be studied by static or by dynamic models. The first one is termed Static Transmission Expansion Planning (STEP) and tries to discover an optimal topology of expansion, i.e, where should be installed an equipment that minimizes the installation and operational costs for a given scenario of generation and load. A dynamic model is more complex and it aims at, besides answering the questions of where, defining when to install the network additions, creating therefore a plan of investment along successive periods of time (Latorre, 2003).

The purpose of STEP is to determine how the transmission capacity of a network should be enlarged, satisfying the increasing demand, i.e, from a pre-existing list of candidate circuits to be built, identify those that will be constructed to minimize the operation cost of the system, supplying the forecasted demand along a planning horizon (Mendonça, 2013). The increasing demand implies modifying the system over time so that the load is properly supplied. There are usually different alternatives to do this that include not only building new transmission lines but also installing new generation facilities closer to demand centers.

In whatever way, it is frequently not economically feasible or even possible to build generating units near the demand centers. On the other hand, transmission expansion can also enable the optimal dispatch of power plants, because having a stronger transmission system provides more flexibility to dispatch generation apart from improving the reliability of the system and decreasing the likelihood of having congested branches.

Solving this optimization problem is an arduous task since it has some special features that increase its complexity: 
- The search space is non-convex so that several solution algorithms may converge to local optima;

- In some cases there are isolated smaller systems;

- The problem typically has an integer nature leading to the phenomenon of combinatorial explosion of investment alternative plans. This requires a high computational effort to identify good quality plans.

These characteristics correspond to the main difficulties in developing high-performance tools in terms of speed, efficiency and robustness to solve the STEP problem. The literature of this area includes a variety of models and tools to solve the STEP and to address the difficulties mentioned above. These models and tools can be organized as follows:

- Classical Optimization Algorithms

They use mathematical decomposition techniques and generally find global optimal solutions for a relaxed version of the original integer problem in which integer investment alternatives are substituted by continuous variables. However, they usually require a large computational effort and therefore they display difficulties to solve medium and large systems as most of real transmission systems are. In some cases they can also show convergence problems as detailed in (Garver, 1970 and Romero, 2005).

- Constructive Heuristics Algorithms (CHAs)

These approaches correspond to simplified procedures that are suitable to identify feasible solutions for complex problems using efficient and easily applied algorithms. They have little computational effort, but rarely do they find the optimal global solution, especially if one is addressing real transmission networks (Rider, 2008 and Silva, 2010).

- Metaheuristics

They are heuristics techniques that are enhanced with particular search procedures in most cases inspired in natural mechanisms. They are especially suited to solve complex and combinatorial problems usually identifying optimal or suboptimal solutions even for large systems. However, they are typically associated to large computational efforts (Michalewicz, 2004).

Given the shortcomings of the above approaches, this paper details a method for solve the STEP problem based on a tool called Simulated Annealing (SA) which in turn is a probabilistic local search technique, and is based on an analogy with thermodynamics. This method will be used in hybrid form with the Gaussian Probability Density Function and their motivations will be detailed in a chapter 2, this hybridization was applied to an academic system known as Garver-6 bus, widely detailed in (Latorre, 2003), and shown to be a potential object of study for real transmission systems, since this application there was the optimal results with low computational effort.

Regarding the structure of the paper, following the introduction already presented, the chapter 2 refers to theory and parameters of Simulated Annealing. In chapter 3 was present a brief introduction to Gaussian Probability Density Function and its use in STEP problem. The chapter 4 shows the application of the SA taking into account the Gaussian Probability Density Function as a decision criterion and the chapter 5 is present the results obtained in application of this new tool in the test system Garver-6-Bus as well a discussion about these results. Finally the chapter 6 refers to conclusions about this research. 


\section{Simulated Annealing}

As already stated, the Simulated Annealing consists of a probabilistic local search technique, based on an analogy with thermodynamics, wherein a current solution is replaced by a next solution, selected according to an objective function and a variable $T$ (actual temperature by analogy). As the bigger $T$, bigger is the random component to be included in the next chosen solution. As the algorithm progresses, the value of $T$ is decremented, starting the algorithm to converge to an optimal solution necessarily local.

SA can be used to solve combinatorial optimization problems. It's formulated as follows (Michalewicz, 2004): Let $G$ be a set of configurations and $v$ the cost associated with each configuration of $G$. The solution to the combinatorial problem consists in finding, in search space, one configuration of $(G, v)$ with lowest cost. The SA algorithm starts with an initial configuration $G o$ and an initial temperature $T=T_{o}$ and generates a sequence of configurations $N=N_{o}$. So the temperature is decreased; Now is determined a new number of steps to be performed at the temperature level and the process is then repeated. A configuration $S_{j}$ is accepted if its cost is less than that of the current configuration $S_{i}$, but, if not, it still can be accepted with a certain probability. This ability to perform uphill moves allows SA to escape from local optimal configurations. The process is controlled by a cooling schedule that determines how the temperature $(T)$ changes during the optimization process. It is important to note that the larger the value of $T$, less will be the significance of the comparison of current and candidate configuration (Carmona, 2009). The figure 1 summarizes the SA algorithm:

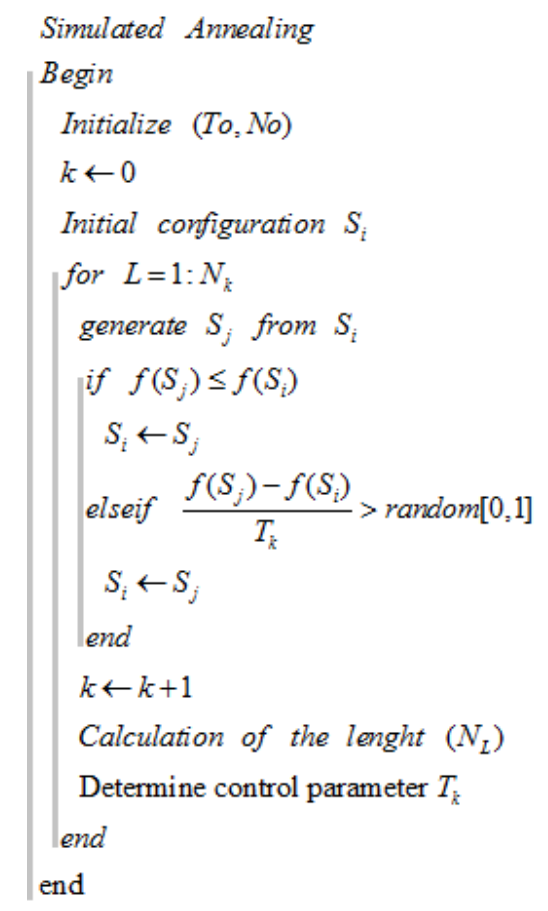

Figure 1: The Simulated Annealing Algorithm

As the search space of STEP problem is non-convex, several solution algorithms may converge to local optima. For instance, in a Local Search Heuristic (LSH), the algorithm basically selects, from a random point in space search, the best neighbor to this point satisfying the constraints of the problem. This algorithm changes the current solution for a neighbor solution (better than that) until the change of in the solution is not more beneficial. 
In maximization problem, for instance, using the $\mathrm{LSH}$, consider function that is show in figure 2 , this function is defined in $t_{o}$ to $t_{f}$ and has a local maximum at $t_{l}$ and the global maximum in $t_{m}$. If the initial solution is in $t_{1}$ it will change and follow the neighborhood until it reaches the local maximum in $t_{l}$, in this case, the solution can't be further modified because any modification in this neighborhood would be worse than the local maximum. But if it would could make changes that deem worse solutions in the neighborhood, for example modifying the optimum location for a solution at $t_{2}$ and so on, perhaps we could achieve the global maximum $t_{m}$ as show in figure 2 :

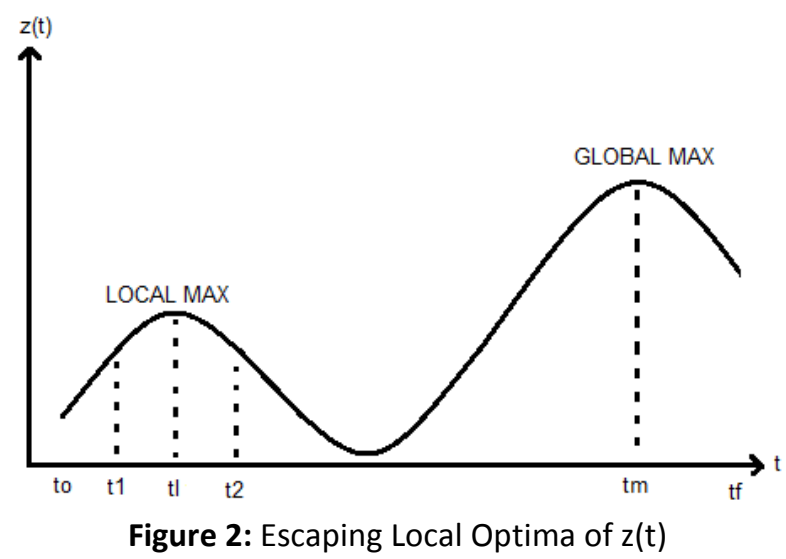

Therefore SA has the ability to accept new solutions worst of the current using the temperature. This is the main feature of the SA, escape from a local optima, however, as the process usually has a number of iterations, can occur in solving the STEP problem a change of current solution to a worse solution close to the end of the process. In order to avoid this "bad exchange" near to the end of the process was used in this study the Gaussian Probability Density Function, which will be described in the next chapter. This function will be adapted to consider this type of change in the beginning of process and go decreasing according to the method draws to a close.

Finally, the cooling schedule is the control strategy of SA and is characterized by following parameters:

- Initial temperature $\left(T_{o}\right)$;

- Final temperature $\left({ }^{T}\right)$;

- Number of transitions at temperature $T_{k}\left(N_{k}\right)$;

- Temperature rate of change $\left(T_{k+1}=g\left(T_{k}\right) \cdot T_{k}\right)$, where $g$ is a function that controls the temperature.

The efficiency and the quality of the algorithm as well as the number of iterations will depend of these parameters. The procedures used in the calculation of parameters are based on the idea of thermal equilibrium and are detailed in chapter 4.

\section{Gaussian Probability Density Function in STEP problem}

Regarding the SA algorithm structure, after creating a neighbor solution, it is compared to the current solution. If the value of the neighboring solution is less than the current solution, the current solution is updated as the neighboring solution. Otherwise the neighboring solution may or may not be discarded depending on a probability parameter. In this paper, 
was used the function of normal probability distribution, which equation follows bellow (Oja, 2000):

$$
p(x)=\frac{1}{\sqrt{2 \pi \sigma^{2}}} \cdot e^{-\frac{(x-\mu)^{2}}{2 \sigma^{2}}}
$$

Where $\mu$ (mean) determines the peak point of the symmetric density, $\sigma$ (standard deviation), its effective width (flatness or sharpness of the peak) and $x$ is the current iteration. The figure 3 bellow represents the Gaussian Probability Density Function with mean $\mu$ and standard deviation $\sigma$ :

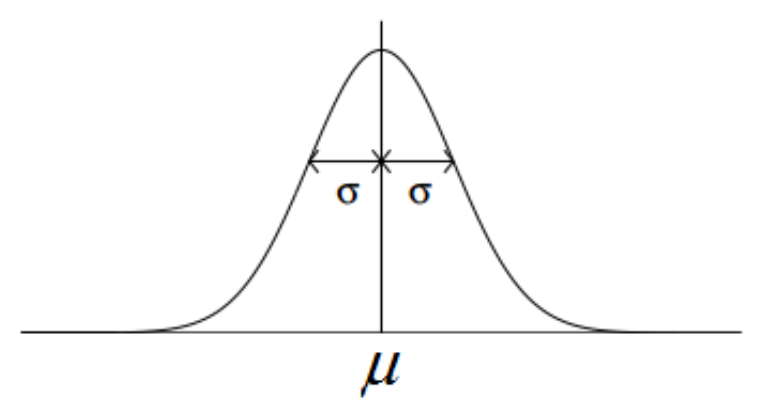

Figure 3: Gaussian Probability Density Function

The probability points are normalized between 0 and 1 as shown in Figure 3. For this paper was used the mean equals to the half number of iterations of the process and the standard deviation equals to a tenth of that value, these values were selected from experiments with the database, in this case Garver-6-Bus, and obtained a better performance.

The number of iterations of the process is obtained from the initial temperature $T_{o}$ and final temperature $T_{f}$ of the process, as well as from temperature rate of change $g\left(T_{k}\right)$, that in this case was used as 0,75 (constant function) and the number $N_{k}$ of transitions at temperature $T_{k}$.

The Gaussian Probability Density Function is used in case of being chosen a neighboring solution that has a higher value than the current solution, is important to clarify that in this hybrid SA the solution never exceeds the limit of viability (as well as in local search), i.e, never becomes unfeasible, the probability function is only used to evaluate the switch between a worse solution but also feasible. The solutions exchange process can be studied in three distinct phases which motivate the use of this function:

I. Phase 1: Natural removal

The process starts with all possible circuits inserted into the initial solution; the initial iterations will naturally remove circuits in order to improve the solution.

II. Phase 2: Swap or Addition

After phase one, will appear essential circuits that if removed will result in the system operate improperly, and therefore, should be a neighbor solution originating swap or addition, and hence, the algorithm should provide a greater likelihood to accept a cost solution greater with the expectation that it will improve in the future.

III. Phase 3: Forced removal

In this phase the removal of circuits is a priority. The probabilities to change for a solution with higher cost decreases over the iterations since the process nears completion and the 
solution should be given. Figure 4 bellow show the phases in the Gaussian Probability Density Curve:

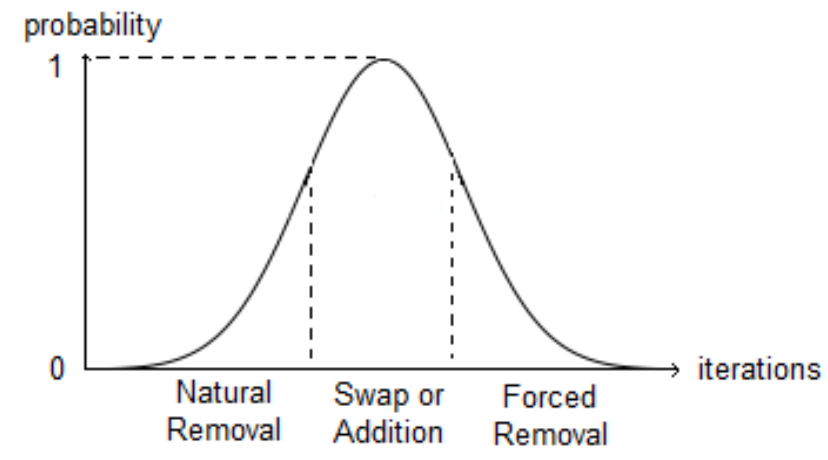

Figure 4: Phases to change for worse solutions

\section{Hybrid SA for STEP problem}

In this section, a Hybrid Simulated Annealing based on a Local Search and using a Gaussian Probability Density Function is used for solving the STEP problem. For that, some parameters shall be submitted:

- Initial Solution

One can determine the initial solution in myriad ways, from the initial topology to the topology with the maximum number of allowed circuits. This process can be done at random or by a pre-determined expression, or even for a tool for rapid convergence with for example CHA. In this paper the initial solution is chosen with the topology relating of all additions permitted into the circuit, i.e. the initial solution contains all the additions allowed. Over the iterations circuits are removed in order to get an acceptable and feasible solution.

- The evaluation function

To evaluate a solution is necessary, in STEP problem, run a linear program in order to determine the corresponding load shedding, which forms part of the cost function. This linear program is described below (Latorre, 2003):

$$
\text { Minimize } v=\sum \alpha_{k} \cdot r_{k}
$$

Subject to:

$$
\begin{gathered}
S . f+g+r=d \\
f_{i j}-\gamma_{i j} \cdot\left(n_{i j}+n_{i j}\right) \cdot\left(\theta_{i}-\theta_{j}\right)=0 \\
\left|f_{i j}\right| \leq\left(\begin{array}{c}
o \\
n_{i j}+n_{i j}
\end{array}\right) \cdot \bar{f}_{i j} \\
0 \leq g_{i} \leq \bar{g}_{i} \\
0 \leq \eta_{i j} \leq \bar{\eta}_{i j} \\
\eta_{i j} \text { integer, } x_{i j} \text { discrete, } f_{i j}, \theta_{i} \text { unrestricted }
\end{gathered}
$$

In this formulation $r$ is the vector of artificial generators, $\alpha$ is a penalty factor for load shedding, $\eta_{i j}$ is the current solution of built circuits from $\mathrm{i}$ to $\mathrm{j}, \mathrm{S}$ is the transposed incidence node-branch matrix of the power system, $f$ is the vector of flows, $g$ is the generation vector whose maximum value is $\bar{g}_{i}, \mathrm{~d}$ is the demand vector, $\gamma_{i j}$ is the susceptance of the circuit, 
$n_{i j}^{o}$ is the number of circuits in the base topology, $\theta$ is the voltage angle of bus and $\bar{f}_{i j}$ is the maximum power flow of a circuit in $\mathrm{i}-\mathrm{j}$.

- Neighborhood structure

In this paper, the neighborhood structure is formed by three phases that result from the current configuration by:

a) Removing a circuit of current topology - phase one;

b) Swapping two circuits (adding and removing two random circuits) - phase two.

c) Adding a new circuit - phase three.

That is, to select a neighbor of the current solution, a circuit is removed and it is checked whether this solution is feasible (phase one), if not, inserts a circuit in another path, and again the feasibility is verified (phase two), if the system still operating improperly (with load shedding) the removed circuit in phase one is reattached as the added circuit in phase two is removed, and after this, is added a new circuit where was tried remove, i.e, on path of phase one. This last added occur because this path is important for the system. If the last circuit is redundant it will be removed in other iteration.

\section{Results and discussions}

SA algorithm was used to find an acceptable solution to the Garver- 6 Bus test system, that is extensively as studied in (Latorre, 2003), with and without reschedule generation. For this system was used the following parameters:

$\alpha=100$

$g\left(T_{k}\right)=0,75$

$N_{k}=90$

$T_{o}=400$

$T_{f}=1$

The number of iteration for these cases is 1890 , these values above were also selected from experiments with the database and obtained better performance.

a) Garver-6-Bus with Reschedule Generation

For this case the algorithm found the solution in 99,63 seconds. The solution has the expansion on the lines 4-6 (3) and 3-5 (1) as shown in figure 6. The cost for this expansion is US\$110.000.000,00 and this solution is the optimal expansion for this circuit (Gomes, 2014).

The new topology of the system with the additions of the lines is shown in Figure 5 :

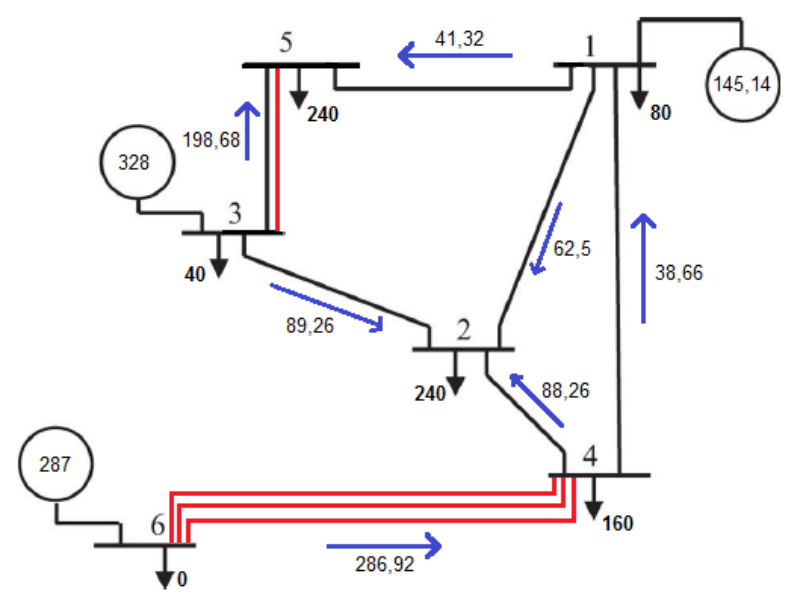

Figure 5: Expansion planning for Garver-6-Bus with reschedule generation 
Throughout the process the cost of the solution was varying according to Figure 6 , in this figure it is evident that the exchange of current solutions to the worst solutions, with higher costs in this case, happen after the iteration number about 400 , that is when the changes for cheaper solutions leave the system operating incorrectly, i.e., cutting load or with congestion on the lines. This process continues until approximately iteration 1600, which is when the process is nearing its end (iteration 1890).

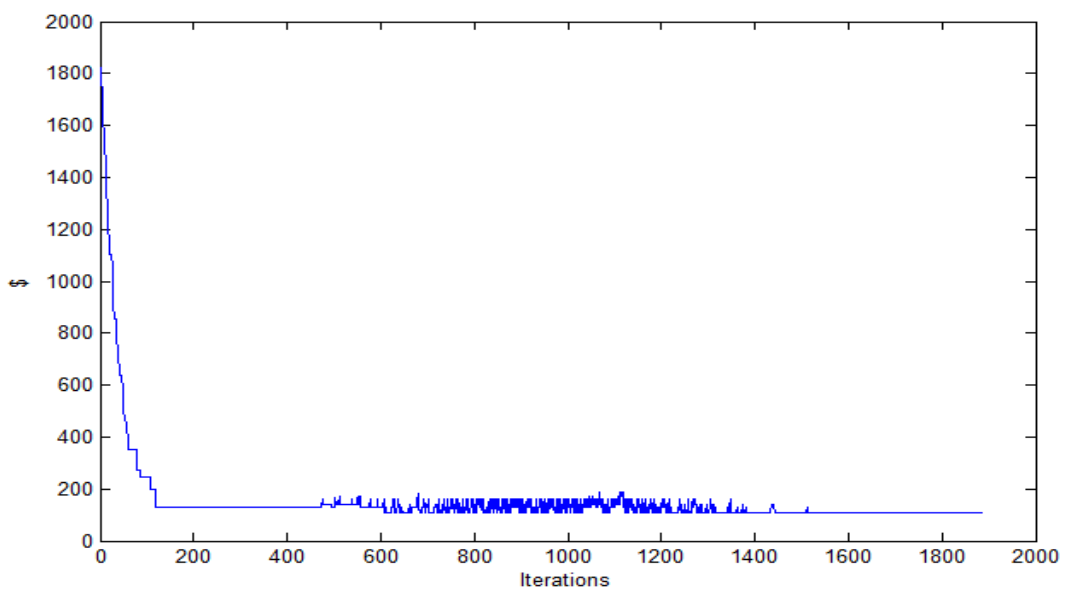

Figure 6: Variation in the cost solution - With reschedule generation

b) Garver-6-Bus without Reschedule Generation

For this case the algorithm found the solution in 228,4 seconds. The solution has the expansion on the lines 4-6 (3), 2-6 (3) and 3-5 (1) as shown in figure 7. The cost for this expansion is US\$200.000.000,00 and this solution is the optimal expansion for this circuit (Gomes, 2014).

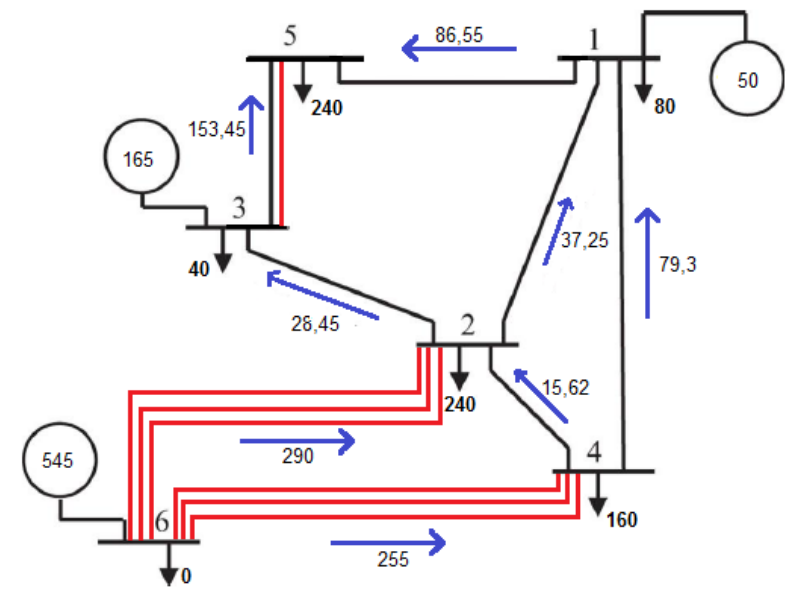

Figure 7: Expansion planning for Garver-6-Bus with reschedule generation

Throughout the process the cost of the solution was varying according to Figure 8 , in this figure one can note that near to iteration 400 the process change the current solution by a worst solution, i.e., with a higher cost. This exchange was essential to the process improve the final solution, since the solution already had converged near 400,000,000.00. 


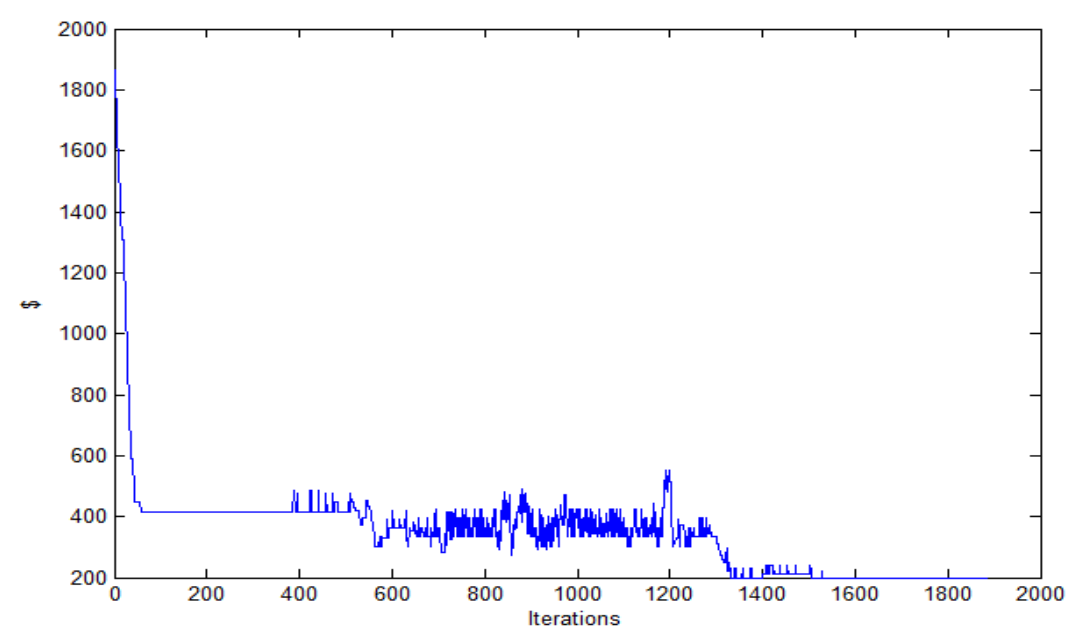

Figure 8: Variation in the cost solution - Without reschedule generation

\section{Conclusions}

In this paper a Simulated Annealing is proposed based in a Local Search and it uses the Gaussian Probability Density Function normalized to accept or not a neighbor solution with a greater cost than the current solution. This method proved to be a powerful tool in which to escape from local optima with relatively low computational effort. Was able to find the optimal expansion for Garver-6-Bus system with and without reschedule generation.

The tool has also proven to be an important subject of study as evidence has proven its effectiveness in regard to removal of extra circuits. The use of Gaussian Probability Density Function as decision support for Simulated Annealing provides greater confidence to escape local optima, since the probabilistic steps exchanges are predetermined. The use of this method for real and large systems can be considered as a future work.

\section{References}

G. Latorre, R. D. Cruz, J. M. Areiza, A. Villegas, "Classification of publications and models on transmission expansion planning", IEEE Transactions on Power Systems, vol. 18, no 2,pp. 938-946, May. 2003.

I. M. Mendonça, C. S. Ivo, A. L. M. Marcato, B. H. Dias "Transmission expansion optimization via constructive heuristic technique", 2013 IEEE Grenoble PowerTech, POWERTECH, p. 15, June 2013.

L. L. Garver, "Transmission Network Estimation Using Linear Programming," IEEE Trans. Power Apparatus and Systems, vol. PAS-89, n 7, pp. 1688-1697, Sep. 1970.

I. G. Sánchez, R. Romero, J. R. S. Mantovani and M. J. RIDER. "Transmission - Expansion Planning Using the DC Model and Nonlinear - Programming Technique". IEE Proceedings of Generation, Transmission and Distribution, vol. 152, no. 6, pp. 763-769, Nov 2005.

M. Rider, A. V. Garcia, R. Romero, "Transmission System Expansion Planning by a Branchand-Bound Algorithm", IET Generation, Transmission \& Distribution, vol. 2, no. 1, pp. 9099, January 2008.

Z. Michalewicz, D. Fogel, How to solve it: Modern heuristic, vol. I. New York: Springer, 2004, p. 194.

M. C. Rocha, J. T. Saraiva, "Muliyear Transmission Expansion Planning Using Discrete Evolutionary Particle Swarm Optimization", in Proc. 17th Power Systems Computation Conf., Stockholm, 2011. 
A. M. L. Silva, L. S. Rezende, L. A. F. Manso, L. C. Resende "Reliability worth applied to transmission expansion planning based on ant colony system", International Journal of Electrical Power \& Energy Systems, vol. 32, no. 10, pp. 1077-1084, Jul. 2010.

M. C. Carmona, R. P. Behnke, "Transmission Network Expansion Planning by a Hybrid Simulated Annealing Algorithm", IEEE 15th International Conference on Intelligent System Applications to Power Systems, 2009.

H. Aapo, K. Juha, E. Oja, Independent Component Analysis, Vol I, New York, WileyInterscience Publication.

P.V. Gomes, "Critical analysis of the main Constructive Heuristic Algorithms used in the static transmission expansion planning," 2014.

\section{Acknowledgment}

The author thanks CAPES Foundation, Ministry of Education of Brazil, for financing this research. 